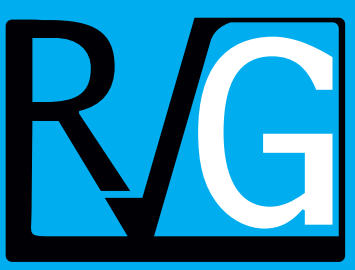

Año 21 No. 74

Abril - Junio 2016

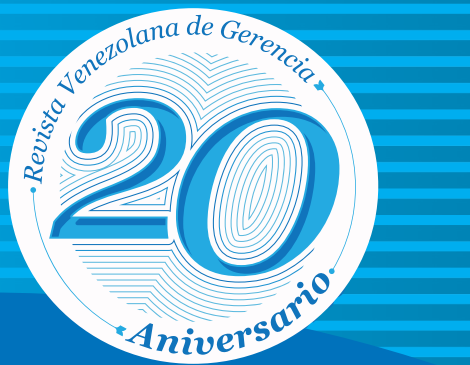

Venezolana de
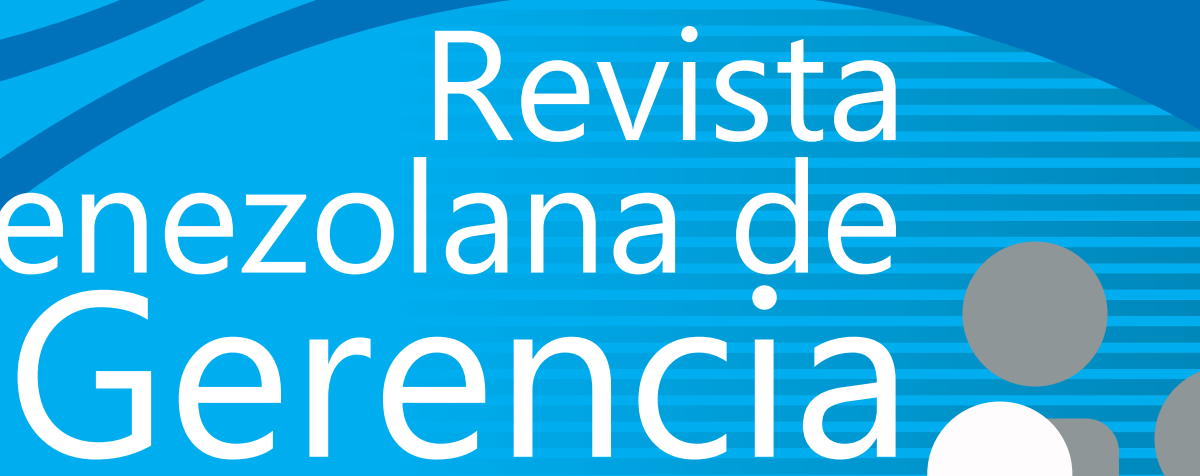


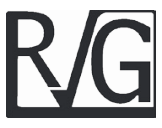

\title{
Innovación tecnológica en microempresas españolas
}

\author{
Pérez Estébanez, Raquel ${ }^{1}$
}

\section{Resumen}

La innovación tecnológica es un proceso fundamental para el desarrollo y crecimiento en un país, más si éste se encuentra en situación económica adversa, como es el caso de España. Es un requisito indispensable para la evolución y pervivencia de las empresas de menor dimensión que sufren en mayor medida una crisis económica como la que ha vivido en Europa. Esta investigación pretende analizar la situación de las microempresas, respecto a la implantación y uso de las Tecnologías de la Información y la Comunicación en un contexto de crisis económica, observando el periodo 2005-2014. Este estudio empírico realiza un análisis multivariante a través de un ANOVA comparando las variables Tecnologías de la Información y la Comunicación con la variable temporal, utilizando la base de datos de la Encuesta de uso de Tecnologías de la Información y la Comunicación y Comercio Electrónico sobre una muestra de 13.540 microempresas. Los resultados muestran claramente que, a pesar de la crisis, las microempresas han aumentado su inversión en TIC y además su esfuerzo ha sido mayor respecto a empresas de mayor dimensión. Las empresas consideran las Tecnologías de Información y Comunicación como un componente fundamental de su gestión y de su planificación estratégica, no importa la dimensión que tengan, y de hecho las empresas más pequeñas son las que en este periodo de grandes dificultades económicas y financieras han hecho una apuesta clara por las Tecnologías de Información y Comunicación acortando la distancia que tenían respecto a las grandes.

Palabras clave: tecnologías de la información y la comunicación; innovación tecnológica; crisis económica; microempresa.

Recibido: 20.10.15 Aceptado: 15.03.16

1 Profesora Contratada Doctora de la Universidad Complutense de Madrid, Madrid, España. Ph. D. y Licenciatura en Económicas y Empresariales en la Universidad Complutense de Madrid. E-Mail: raperez@ucm.es 


\title{
Technological innovation in Spanish microenterprises
}

\begin{abstract}
Technological innovation is basic to the development and growth status in a country, more so if it is in adverse economic situation -one seen in Spain. And it is a prerequisite for the evolution and survival of smaller companies that suffer the worst economic adverse situation as it happens now in some European countries. This research aims to analyze whether the economic and financial crisis has influenced the decision of Spanish microenterprises in the implementation and use of Information Technology and Communication, in the period 2005-2014 through a multivariate analysis, ANOVA. This empirical study uses the database of the Survey on Information Technology and Communication usage and E-Commerce on a sample of 13,540 microenterprises. The results demonstrate that, despite the crisis, micro enterprises have increased their use of Information Technology and Communication and also their effort has been greater than in larger companies.
\end{abstract}

Keywords: information and communication Technologies; technological innovation; economic crisis; microenterprise.

\section{Introducción}

En el año 2008 se produce el estallido de la crisis financiera en los mercados internacionales originada en Estados Unidos que afectó a todas las economías en mayor o menor medida; y principalmente a las economías de la mayor parte de los países desarrollados. España ha sido uno de los países más afectados porque la situación se ha visto agravada además por los problemas derivados de su propio modelo económico, sustentado sobre todo en un crecimiento desmedido del sector de la construcción, conocido como "burbuja inmobiliaria" que ha originado un fuerte desempleo en el sector y que, unido a la crisis financiera ha sumido al país en una profunda crisis económica.

En esta difícil realidad las empresas tienen que realizar importantes ajustes y nuevos planteamientos para intentar salir de la situación en que se han visto involucradas y en ese nuevo escenario es donde entran las
Tecnologías de la Información y la Comunicación (TIC), dado que siguen siendo el principal catalizador de la dinámica de innovación de la economía mundial. El nuevo espectro económico en el mundo y los nuevos retos hacen que las empresas se apoyen en las TIC para cubrir demandas de flexibilidad e incremento de productividad (Santana y Aspilcueta, 2015:685), siendo un gran incentivo con el que llegar a un mejor rendimiento y a conseguir reducir los obstáculos financieros y poder acceder a los mercados de capitales (Galia y Legros, 2005).

Las TIC favorecen una organización más eficiente de las estructuras internas empresariales, consiguiendo reducir el coste de mantenimiento de las aplicaciones y aprovechar soluciones identificadas localmente (Rhee, 2005) y facilitando la coordinación entre unidades, lo que mejora la eficiencia en los procesos productivos (Argyres, 1999) y además posibilitan 
la apertura a nuevos mercados y posibilidades de negocio (García-Canal et al, 2008).

Numerosos estudios avalan la teoría de que un uso adecuado de TIC aumenta la productividad, influyendo decisivamente en la riqueza y crecimiento de un país (Brynjolffson et al, 1994 y Dozier y Chang, 2006).

En España, donde el tejido empresarial es tradicionalmente de pequeña dimensión, el uso de las TIC es un requisito imprescindible para el desarrollo económico y el crecimiento (López Sánchez, 2004). En la actualidad, en España las microempresas representan el $95.8 \%$ del tejido empresarial, las pequeñas empresas el 3.5\%, las medianas el $0.5 \%$ y las grandes sólo el $0.2 \%$ (DIRCE, 2015). Esto da una idea de la importancia que tiene la pequeña y mediana empresa en el tejido empresarial español.

Por otra parte, es incuestionable que las TIC se han convertido en instrumentos estratégicos para los negocios pues les permite mantenerse en mercados cada vez más abiertos y competitivos, y es un hecho que para que las pymes puedan lograr ventajas competitivas sostenibles, tienen que reconocer la importancia de las TIC en sus organizaciones. En un entorno económico muy complejo y con un escenario desfavorable de los cinco últimos años, ha crecido el número de pymes españolas que han adoptado y utilizan las TIC como herramientas básicas para optimizar la gestión interna, incrementar su productividad y conseguir una mejora de su posición en el mercado así parece indicarlo el informe ePyme14 publicado por Fundetec, en 2014, pues el $99.2 \%$ de las pymes y grandes empresas disponen ya de esta infraestructura básica (Fundetec Informe ePyme14, 2014). Además, como señala Gimeno (2014:33-44), a partir de 2010 el acceso y uso de las TIC en España ha avanzado al mismo ritmo que el resto de países de la Unión Europea.

Cuanto menor es el tamaño de una empresa, mayor capacidad tiene de adaptarse a los cambios en el entorno (Barney, 1991; Dubois, 1993; Nieto y Fernández, 2006) y esto en la situación de crisis actual es una ventaja clara respecto a las grandes empresas, aunque en su contra tienen grandes dificultades a la hora de obtener recursos. La inversión en TIC es uno de los condicionantes para mejorar el rendimiento del negocio, superar los obstáculos financieros y de ese modo tener acceso a los mercados de valores (Galia y Legros, 2005) por lo que es un requisito imprescindible para las empresas pequeñas si quieren sobrevivir en un entorno hostil. En España, al igual que en Latinoamérica, las pequeñas empresas tienden a la adquisición de tecnología y las mejoras continua, aunque prestan menor atención a la I+D+l (Salazar, Arzola y Pérez, 2010). Una implantación óptima de las TIC en las microempresas (Castillo y García, 2006), (Davis y Sun, 2006) favorece la adaptación a un entorno cambiante porque, a través de los portales de internet, páginas web, etc., se optimizan las relaciones empresariales a larga distancia con clientes extranjeros, se mejora la competitividad y por ende se favorece un mayor dinamismo en la empresa y mayores posibilidades de crecimiento.

Las microempresas necesitan invertir en tecnología que fomente la información y comunicación en distintas áreas de la organización directamente relacionadas con la ganancia o pérdida de cuota de mercado como la producción, la gestión comercial o la innovación, lo que tendrá su reflejo en los resultados y en la productividad obtenida. Muchos autores han estudiado la potencial contribución de las TIC al aumento de la productividad de las empresa pequeñas, 
que aún en época de crisis como ésta, apuestan por la inversión de TIC como estrategia competitiva (Cramm, 2008).

Por todo ello esta investigación se llevó a cabo analizando los principales indicadores clave de Tecnologías de la Información y Comunicación según el Partnership en Medición de TIC para el Desarrollo², que miden el grado de incorporación de las tecnologías de la información en la empresa durante el periodo 2005-2014. Este periodo ha sido escogido porque comienza con un año de auge y crecimiento económico que cambia a partir de 2007 con el estallido de la burbuja inmobiliaria dejando a España desde entonces en una grave crisis económica y termina en la fase de recuperación económica.

El objetivo es investigar si, en esta coyuntura económica y financiera tan adversa, las microempresas han hecho un esfuerzo en este ámbito de innovación tecnológica como herramienta necesaria para su supervivencia y desarrollo. La investigación se justifica porque pretende apoyar la innovación tecnológica en las empresas de menor tamaño, microempresas, como motor de crecimiento económico y competitividad. $\mathrm{Y}$ este ha sido el objetivo principal de la investigación, porque obtener información sobre el uso de las TIC y sobre el acceso a ellas por parte de las empresas es crucial para formular políticas y estrategias concernientes al crecimiento posibilitado por dichas tecnologías, favorecer la cohesión y la inclusión social, y efectuar el seguimiento y la evaluación de los efectos de esas tecnologías en la evolución económica y social.

\section{Las TIC en las empresas en un marco de inestabilidad}

Que las TIC son hoy en día muy importantes en las empresas es un hecho incuestionable que avala una extensa literatura sobre el tema. Está ampliamente demostrado que la tecnología y los avances tecnológicos son un componente clave de la innovación y el crecimiento económico (van Hemert y Nijkamp, 2010) y son bien conocidos los beneficios de las TIC para una empresa en cuanto a ahorro de insumos, reducción general en los costes o mayor flexibilidad y mejoras en la calidad del producto (Arvanitis y Loukis, 2009). Hay estudios que muestran resultados positivos sobre el impacto de las TIC en la eficiencia (Ayed -Mouelhi, 2009). Y según el informe del Banco Mundial sobre tendencias y políticas globales sobre TIC (World Bank, 2006:4), las empresas que utilizan las TIC crecen más rápido, invierten más, y son más productivas y rentables que aquellas que no las usan.

EI uso adecuado de TIC aumenta la productividad de tal modo que tiene una influencia decisiva sobre la riqueza de un país y el crecimiento. Las TIC son consideradas como "una herramienta que impulsa y fortalece la competitividad, incrementando la productividad, disminuyendo los costos y por ende aumentando el crecimiento de las empresas" (Ríos, López y Contreras, 2013). Según la OCDE, los países con mayor inversión de TIC también tienen los mejores índices de crecimiento de la productividad (OCDE, 2000). Sin embargo, el desarrollo con éxito en la sociedad de la información no reside

2 véase http://measuring-ict.unctad.org. 
tanto en la disponibilidad de una buena infraestructura tecnológica como en la mejora de la disposición de la empresa hacia el uso de TIC (Scapens y Jazayeri, 2003), (Scapens, Jazayeri, y Scapens, 1998). La contribución de las TIC a un impacto en resultados es de vital importancia y es por eso que en la mayoría de los países desarrollados y en desarrollo las empresas hacen grandes inversiones para adquirirlas y utilizarlas.

A pesar de la paradoja de la productividad de las TIC (Brynjolfsson y Hitt, 1996), hay estudios recientes que encuentran un efecto positivo estadísticamente significativo de las TIC sobre los resultados empresariales en las empresas de Europa, en Alemania (Bertchek y Kaiser, 2001; Wolf y Zwick, 2002; Hempell, 2003), Suiza (Arvanitis, 2005), España (Pérez, Urquía y Muñoz, 2010) o Reino Unido (Crespi, Criscuolo y Haskel, 2006).

Si analizamos la adopción de TIC en función del tamaño de la empresa, ésta se ha llevado a cabo a un ritmo más rápido en las grandes empresas que en las de menor dimensión, porque las pequeñas empresas tienen menos recursos financieros, menor experiencia técnica y capacidad de gestión, y han comenzado a utilizar más tarde las TIC (Caldeira y Ward, 2003). A pesar de este desequilibrio, según un estudio global de análisis de la evolución de la adopción de TIC en 20 países y sus repercusiones en las empresas (Red BIT Global Research) esta distancia se ha reducido de manera constante en el periodo 2007-2010.

Las ventajas de una óptima utilización de TIC en las microempresas son una mejor adaptación a un entorno cambiante, una mejora en la gestión administrativa y en los procesos internos de fabricación, la posibilidad de nuevos negocios en red, así como, la mejora de las relaciones externas de la empresa y con los clientes o la posibilidad de utilizar la red como un nuevo canal de venta (Garengo, Nudurupati y Bitici, 2007, Castillo y García, 2006, Dozier y Chang, 2006). Las TIC pueden ofrecer a las microempresas información relevante para la toma de decisiones que pueda hacer frente a un mayor grado de incertidumbre en el mercado competitivo (El Louadi, 1998) por lo que éstas necesitan mejorar sus sistemas y capacidad de procesamiento para igualarlas a sus necesidades de información (Van de Ven, Drazin, 1985).

Finalmente hay autores que apuestan por que las empresas aprovechen esta situación de crisis y la transformen en una oportunidad (Clair y Dufresne, 2007). En situaciones de inestabilidad o de dificultades económicas donde deben producirse cambios sustanciales hay empresas que son capaces de mantener sus ventajas competitivas (Abernathy y Clark, 1985; Tushman y Anderson, 1986) y las TIC pueden ser una herramienta que incremente la capacidad de una empresa de obtenerlas (Ravichandran, 2005).

\section{3. ¿Han apostado las microempresas por las TIC en este contexto de crisis?}

Para analizar la implantación y el uso de las Tecnologías de la Información y de la Comunicación en el sector empresarial español en el periodo 20052014 y en concreto en las empresas de menor dimensión, se han obtenido los datos de la Encuesta de uso de TIC y Comercio Electrónico (ETICCE) del Directorio Central de Empresas (DIRCE). Esto ha permitido tener una muestra muy grande y contrastada de empresas que permite hacer análisis relevantes sobre 
la implantación de la Sociedad de la Información en las empresas españolas.

El número total de empresas analizadas ha sido de 13.514 microempresas. El periodo estadístico no puede ser ampliado porque es en el año 2004 cuando se realiza por el DIRCE el primer estudio piloto sobre las microempresas y no ofrece información sobre todas las variables principales objeto del estudio, por lo que se comienza en el 2005 , año relevante por ser un periodo de crecimiento económico y la muestra termina en el año 2014, por lo que es el último año de estudio en este artículo, también relevante porque es un año de recuperación económica.

Para poder hacer la investigación comparativa se ha restringido el número de variables a siete, que definen el grado de uso de TIC en el periodo 2005-2014 consideradas indicadores clave de TIC según el Partnership en Medición de TIC para el Desarrollo (Cuadro 1).

\section{Cuadro 1}

\section{Principales variables TIC}

\begin{tabular}{cc}
\hline Variable & \multicolumn{1}{c}{ Descripción } \\
\hline TIC1 & $\%$ Empresas que disponían de ordenadores \\
TIC2 & $\%$ Empresas que disponían de Red de Área Local \\
TIC3 & $\%$ Empresas que disponían de conexión a Internet \\
TIC4 & $\%$ Empresas que disponían de conexión a Telefonía móvil \\
TIC5 & $\%$ Empresas que disponían de correo electrónico (e-mail) \\
TIC6 & $\%$ Empresas que disponían de conexión a Banda ancha \\
TIC7 & $\%$ Empresas que disponían de conexión a Internet y sitio/página web
\end{tabular}

Fuente: Elaboración propia.

Los resultados obtenidos del total de empresas en los años analizados se exponen a continuación. En primer lugar se obtienen los estadísticos descriptivos y las correlaciones (Tabla 1) que como se aprecia hay una correlación entre las variables TIC del estudio significativa; y posteriormente se realiza un análisis comparativo de las medias de las variables a través de un análisis multivariante de la varianza, ANOVA para ver si la variable independiente o factor temporal (2005-2014) ha influido o no en las variables dependientes, es decir, si las microempresas han seguido incorporando las TIC en sus estrategias de negocio y su gestión a pesar de la crisis económica.

La hipótesis que se pone a prueba en el ANOVA es que las medias poblacionales son iguales, y si esto es así, significa que los grupos no difieren en la variable dependiente y por lo tanto el factor es independiente de la variable dependiente. El estadístico $\mathrm{F}$ refleja el grado de parecido entre las medias comparadas, de tal forma que si el nivel crítico asociado a $\mathrm{F}$ es menor que 0,05 , se rechaza la hipótesis de igualdad de medias y por lo tanto las medias poblacionales comparadas no son iguales. 
Tabla 1

Estadísticos descriptivos y matriz de correlaciones

\begin{tabular}{|c|c|c|c|c|c|c|c|c|c|}
\hline \multirow[t]{2}{*}{ Variable } & \multirow[t]{2}{*}{ Media } & \multirow[t]{2}{*}{ Desv. Típ. } & \multicolumn{7}{|c|}{ Correlaciones } \\
\hline & & & TIC1 & TIC2 & TIC3 & TIC4 & TIC5 & TIC6 & TIC7 \\
\hline TIC1 & 64,8129 & 7,40794 & & & & & & & \\
\hline TIC2 & 20,9447 & 4,52581 &, $643^{* *}$ & & & & & & \\
\hline TIC3 & 56,3194 & 9,70516 &, $922^{* *}$ &, $657^{* *}$ & & & & & \\
\hline TIC4 & 69,6499 & 6,32998 &, $602^{* *}$ &, $257^{* *}$ &, $519^{* *}$ & & & & \\
\hline TIC5 & 62,347 & 15,85651 & $-0,099$ & 0,082 &,$- 260^{* *}$ &, $176^{*}$ & & & \\
\hline TIC6 & 82,7608 & 21,53183 &, $501^{* *}$ &, $306^{* *}$ & ,669** & 0,128 &,$- 857^{* *}$ & & \\
\hline TIC7 & 23,0285 & 6,07901 &, $635^{\star *}$ &, $420^{* *}$ &, $636^{* *}$ &, $508^{* *}$ & $-0,116$ & $398^{* *}$ & \\
\hline
\end{tabular}

* P-valor < 0,1; ** P-valor < 0,05; ${ }^{* * *}$; P-valor < 0,01.

Fuente: Elaboración propia.

Los resultados del análisis (Tabla

2) son estadísticamente significativos al $99 \%$ para todas las variables, por lo que podemos decir que la variable independiente ha influido en la adopción y uso de TIC en las microempresas, es decir las medias poblacionales no son iguales por lo que rechazamos la hipótesis nula. Además, a pesar de las difíciles circunstancias esa relación ha sido positiva, tal y como se observa, los valores de F son positivos, ha aumentado en este periodo el número de microempresas que disponen de ordenador $(\mathrm{TIC} 1=11,326)$, igualmente aunque en menor proporción han aumentado aquellas que disponen de Red de Área Local (TIC2 $=6,046)$, también han sufrido un aumento las microempresas que tienen conexión a Internet $(\mathrm{TIC} 3=36,142)$, las que disponen de conexión a Telefonía móvil también se incrementan $(\mathrm{TIC} 4=7,839)$ y las que soportan un mayor incremento han sido tanto en el número de microempresas que poseen banda ancha (TIC6 $=767,751)$ como las que usan el de correo electrónico (e-mail) (TIC5= 138,210) y finalmente aquellas con conexión a Internet y sitio/ página web $(\mathrm{TIC} 7=8,812)$, por lo que se puede concluir que aún en la etapa de crisis económica, las microempresas han aumentado su nivel de uso y adopción de tecnologías de la información a pesar del coste que conlleva.

En el análisis de los datos de la serie temporal 2005-2014 se puede observar (Gráfico 1) que en el caso de las variables $\mathrm{TIC}$, tanto las relacionadas con el grado de incorporación de tecnologías informáticas, como las derivadas del uso en su actividad o las relacionadas con la utilización de otras TIC la tendencia es creciente. Sin embargo, hay que hacer un matiz en el caso del uso de la telefonía 


\section{Tabla 2}

Relación entre las variables TIC y la variable temporal

\begin{tabular}{ccccccc}
\hline & & $\begin{array}{c}\text { Suma de } \\
\text { cuadrados }\end{array}$ & gl & $\begin{array}{c}\text { Media } \\
\text { cuadrática }\end{array}$ & F & Sig. \\
\hline \multirow{2}{*}{ TIC1 } & Entre grupos & 3696,177 & 9 & 410,686 & 11,326 &, 000 \\
& Dentro de grupos & 6236,664 & 172 & 36,260 & & \\
& Total & 9932,841 & 181 & & & \\
TIC2 & Entre grupos & 891,003 & 9 & 99,000 & 6,046 &, 000 \\
& Dentro de grupos & 2816,404 & 172 & 16,374 & & \\
& Total & 3707,408 & 181 & & & \\
TIC3 & Entre grupos & 11151,702 & 9 & 1239,078 & 36,142 &, 000 \\
& Dentro de grupos & 5896,707 & 172 & 34,283 & & \\
& Total & 17048,409 & 181 & & & \\
TIC4 & Entre grupos & 2109,462 & 9 & 234,385 & 7,839 &, 000 \\
& Dentro de grupos & 5142,958 & 172 & 29,901 & & \\
& Total & 7252,420 & 181 & & & \\
TIC5 & Entre grupos & 31523,060 & 7 & 4503,294 & 138,210 &, 000 \\
& Dentro de grupos & 4431,291 & 136 & 32,583 & & \\
& Total & 35954,351 & 143 & & & \\
& Entre grupos & 81877,083 & 9 & 9097,454 & 767,751 &, 000 \\
TIC6 & Dentro de grupos & 2038,112 & 172 & 11,849 & & \\
& Total & 83915,194 & 181 & & & \\
& Entre grupos & 2110,842 & 9 & 234,538 & 8,812 &, 000 \\
TIC7 & Dentro de grupos & 4577,906 & 172 & 26,616 & & \\
& Total & 6688,748 & 181 & & & \\
\hline
\end{tabular}

Fuente: Elaboración propia.

móvil, porque entre el año 2007 y el 2009 sufre un decrecimiento, seguramente motivado por la agudización de la crisis económica y por tanto la reducción de la actividad económica dado que se cerraron numeras empresas y negocios, sobre todo, de autónomos y microempresas; aunque cambia nuevamente su tendencia a partir del año 2010. Tal y como Heeks (2010) y Ojo, Janowski y Awotwi (2013) afirman en sus investigaciones, el uso del teléfono móvil es un factor altamente determinante del desarrollo económico.

En estos diez años ha aumentado el número de microempresas que han utilizado las TIC en sus negocios y en línea con Bollou and Ngwenyama (2008) y Oliveira y Fraga (2011), la adopción y uso de TIC ha sido un motor del crecimiento económico local. 


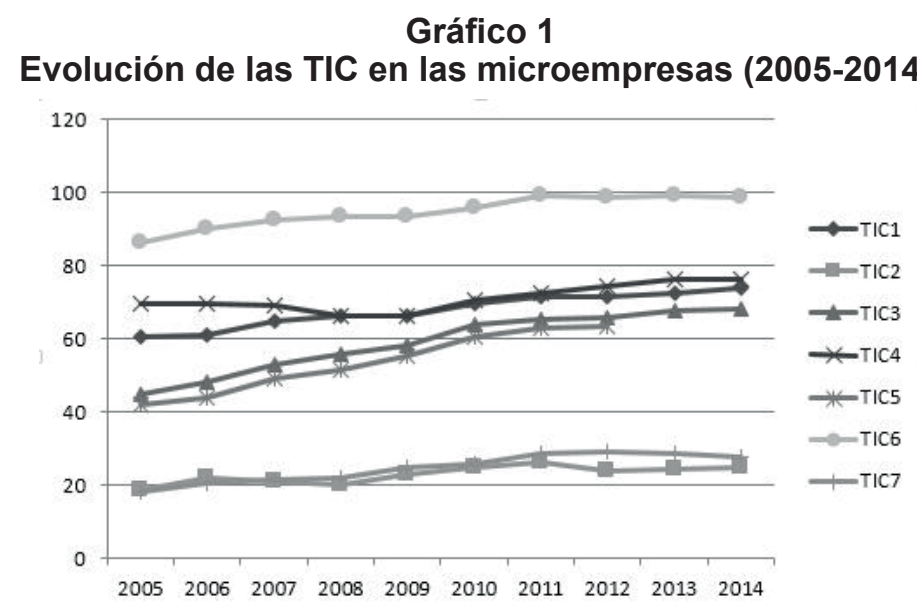

Fuente: Elaboración propia según datos de la Encuesta de uso de TIC y Comercio Electrónico (ETICCE). INEbase.

Si se hace una comparativa por tamaño de empresa para ver cómo están situadas las microempresas respecto al resto de empresas en los años extremos de esta serie temporal, 2005 y 2014, se puede ver claramente en el Gráfico 2 que el esfuerzo en TIC ha sido mayor en las microempresas que en el resto de empresas españolas. El incremento medio en TIC de este periodo ha sido del 13,34\% en las microempresas, $10 \%$ en pequeñas, $5,6 \%$ en medianas y sólo el $2,64 \%$ en las de mayor dimensión. Este esfuerzo ha sido notable en cuanto al aumento en el número de microempresas con conexión a internet además de banda ancha, que disponen de correo electrónico y que utilizan tanto internet como el correo electrónico en sus negocios.

Gráfico 2

\% Variación de TIC por tamaño de empresas (2005 y 2014)

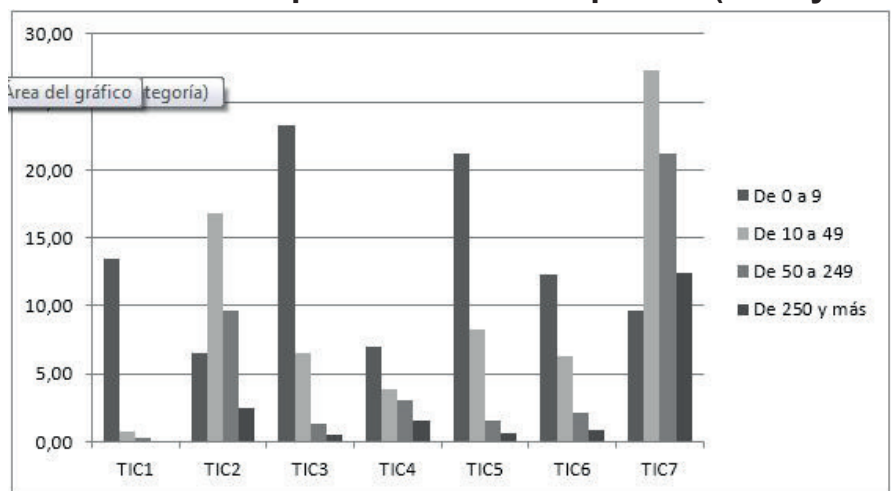

Fuente: Elaboración propia según datos de la Encuesta de uso de TIC y Comercio Electrónico (ETICCE). INEbase. 
Profundizando más, se puede ver en la Tabla 3 que, por un lado en el año 2005, que fue un año de auge económico, las microempresas ocupan el último lugar en cuanto a su incorporación a la sociedad de la información comparadas con las Pymes y grandes empresas. Pero, si se atiende al año 2014 aunque sigue habiendo distancia, ésta claramente se ha acortado. Es decir, durante los últimos años las microempresas han hecho una apuesta clara por las TIC, debido a que han sido cada vez más conscientes de las posibilidades y ventajas que pueden ofrecerles desde un punto de vista estratégico y organizativo porque les posibilitan una mejora interna en la gestión, les facilitan nuevas posibilidades de negocios y les abren las puertas a nuevos mercados a nivel mundial a un coste relativamente bajo.

Tabla 3

Comparativa de las principales variables TIC por tamaño de empresas (2005-2014).

\begin{tabular}{ccccccccc}
\hline & \multicolumn{2}{c}{ De 0 a 9 } & \multicolumn{2}{c}{ De 10 a 49 } & \multicolumn{2}{c}{ De 50 a 249 } & \multicolumn{2}{c}{ De 250 y más } \\
\hline & $\mathbf{2 0 0 5}$ & $\mathbf{2 0 1 4}$ & $\mathbf{2 0 0 5}$ & $\mathbf{2 0 1 4}$ & $\mathbf{2 0 0 5}$ & $\mathbf{2 0 1 4}$ & $\mathbf{2 0 0 5}$ & $\mathbf{2 0 1 4}$ \\
\hline TIC1 & 60,6 & 74,1 & 98,3 & 99,07 & 99,3 & 99,56 & 99,8 & 99,84 \\
TIC2 & 18,6 & 25,1 & 68,0 & 84,85 & 86,2 & 95,81 & 95,4 & 97,95 \\
TIC3 & 44,8 & 68,0 & 91,7 & 98,23 & 98,2 & 99,5 & 99,4 & 99,84 \\
TIC4 & 69,5 & 76,5 & 91,7 & 95,58 & 95,3 & 98,38 & 97,7 & 99,3 \\
TIC5 & 42,0 & $63,2^{*}$ & 89,3 & $97,5^{*}$ & 97,5 & $99^{*}$ & 99,3 & $99,9^{*}$ \\
TIC6 & 86,3 & 98,6 & 93,3 & 99,64 & 97,7 & 99,87 & 99,1 & 100 \\
TIC7 & 18,1 & 27,8 & 46,6 & 73,97 & 68,2 & 89,4 & 82,9 & 95,28 \\
\hline
\end{tabular}

*último año disponible 2012

Fuente. Elaboración propia según datos de la Encuesta de uso de TIC y Comercio Electrónico (ETICCE). INEbase.

\section{Consideraciones finales}

La coyuntura económica española actual, con una fuerte crisis económica motivada fundamentalmente por la crisis financiera mundial y agravada por la burbuja inmobiliaria, ha dejado en una situación muy complicada a las empresas españolas. Sobre todo a partir de 2008 el considerable aumento del desempleo, que las empresas no han tenido capacidad de absorber, y la disminución de la producción y por ende del consumo, así como las serias dificultades a la hora de conseguir crédito las han obligado a plantearse nuevas estrategias de negocio. 
En este panorama, las empresas han seguido apostando por las tecnologías de la información como una herramienta fundamental de sus estrategias empresariales y de su capacidad para alcanzar nuevas cotas de mercado. $Y$ de entre todas las empresas destacan las microempresas por el enorme esfuerzo que han hecho en la adopción y uso de TIC en este periodo.

A tenor de los resultados del estudio empírico, hay una relación directa entre el aumento del porcentaje de microempresas que han adoptado y usan las principales TIC y el periodo temporal analizado, que parte de una situación de auge económico, continúa con una etapa de fuerte crisis económica y culmina con el comienzo de la recuperación económica. Las microempresas han sido capaces de acortar las distancias en TIC respecto al resto de empresas y eso, a pesar de las limitaciones en la obtención de financiación, que es mayor cuanto menor es la dimensión de las empresas. Las microempresas no sólo no han reducido sus recursos en la inversión en TIC sino que claramente los han aumentado.

Para concluir, parece claro que las empresas consideran las TIC como un componente fundamental de su gestión y de su planificación estratégica, no importa la dimensión que tengan, y de hecho las empresas más pequeñas son las que en este periodo de grandes dificultades económicas y financieras han hecho una apuesta clara por las TIC acortando la distancia que tenían respecto a las grandes.

Este estudio plantea limitaciones derivadas de la propia muestra que aunque la base de datos utilizada permite que sea muy representativa de la realidad empresarial, sus características limitan el tipo de análisis estadístico que se puede alcanzar.
Futuras líneas de investigación se abren con el desarrollo de nuevos recursos tecnológicos como el cloud computing o la inteligencia de negocio que permitirán el acceso a mejores y más eficientes herramientas Tic con muy poco coste, inaccesibles hasta hace bien poco para las empresas más pequeñas, que les permitirá una mayor y mejor gestión de la información y una más potente capacidad de presencia en el mercado global.

\section{Referencias bibliográficas}

Abernathy, William y Clark, Kim, (1985), Innovation: Mapping the winds of creative destruction, Research Policy, Año 14, pp. 3-22.

Argyres, Nick, (1999), The impact of Information Technology on Co-ordination: Evidence from the B-2 Bomber, Organization Science, Año 10, No 2, pp. 162-180.

Arvanitis, Spyros, (2005), Computerization, workplace organization, skilled labour and firm productivity: evidence for the Swiss business sector, Economics of Innovation and New Technology, Año 14, No 4, pp. 225-249.

Arvanitis, Spyros y Loukis, Euripidis, (2009), Information and communication technologies, human capital, workplace organization and labor productivity: A comparative study based on firmlevel data for Greece and Switzerland, Information Economics and Policy, Año 21, pp. 43-61.

Ayed-Mouelhi, Rim Ben, (2009), Impact of the adoption of information and communication technologies on firm efficiency in the Tunisian manufacturing sector, Economic Modelling, Año 26, pp. 961-967.

Barney, Jay. (1991), Firm resources and sustained competitive advantage, Journal of management, Año 17, No 1, pp. 99-120.

Bollou, Felix y Ngwenyama, Ojelanki, (2008), Are ICT Investments Paying Off in Africa? 
An Analysis of Total Factor Productivity in Six West African Countries from 1995 to 2002. Information Technology for Development, Año 14, No 4, pp. 294307.

Brynjolfsson, Erik y Hitt, Lorin, (1996), Paradox lost? Firm level evidence on the returns to information systems spends, Management Science, Año 42, No 4, pp. 541-558.

Caldeira, Mario M. y Ward, John M, (2003), Using resource-based theory to interpret the successful adoption and use of information systems and technology in manufacturing small and medium sized enterprises, European Journal of Information Systems, Año 12 , pp. 127-141

Castillo, Antonio y García Dotor, María Dolores, (2006), TIC y PYME: Buenas Prácticas para el desarrollo productivo del país, E- Deusto, Spain.

Cramm, Susan, (2008), Smaller IT Budget? Pursue Value Driven development, Harvard Business Review, November 2008.

Crespi, Gustavo, Criscuolo, Chiara y Haskel, Jonathan, (2006), Information Technology, Organizational Change and Productivity Growth: Evidence form UK Firms, Queen Mary College, University of London Working Paper No 558, London.

Davis, Charles H. y Sun, Elaine, (2006), Business Development in Information Technology SMEs in a regional economy: An explanatory study, Journal of Technology Transfer, Año 31, pp. 145-161.

DIRCE, (2015), Directorio Central de Empresas: explotación estadística. Recuperado el 1 de mayo de 2016, de http://www.ine.es/prensa/np794.pdf

Dozier, Ken y Chang, David, (2006), The effect of company size on the productivity impact of Information Technology Investments, Journal of Information Technology Theory and Application, Año 8, No 1, pp. 33-47.

Dubois, David, (1993), Competency-based performance improvement: A strategy for organizational change. HRD Press, Inc, Amherst, MA.
El Louadi, Mohamed, (1998), The relationship among organisation structure, information technology and information processing in small Canadian firms, Canadian Journal of Administration Science, Año 15, No 2, pp. 99-180.

Fundetec Informe ePyme 14 (2014) Análisis sectorial de la implantación de las TIC en la pyme española. Recuperado el 27 de diciembre de 2015, de http://www.ipyme.org/Publicaciones/ InformePyme2014.pdf.

Galia, Fabrice y Legros, Diègo, (2005), Research and Development, Innovation, Training, Quality \& Profitability: Evidence from France, 2ermes-umr-7017-cnrs, Universite Pantheon -Assas Paris II.

García-Canal, Esteban, Rialp-Criado, Alex y Rialp-Criado, Josep, (2008), Unificación de las TIC y procesos de integración en las fusiones y adquisición horizontales, Universia Business Review, Tercer Trimestre, pp. 25-41.

Gimeno, Manuel, (2014), eEspaña, Informe anual 2014 sobre el desarrollo de la sociedad de la información en España. Fundación Orange, Omán Impresores.

Heeks, Richard, (2010), Development 2.0: the ITenabled transformation of international development. Communications of the ACM, Año 53, No 4, pp. 22-24.

van Hemert, Patricia y Nijkamp, Peter, (2010), Knowledge investments, business R\&D and innovativeness of countries: A qualitative meta-analytic comparison, Technological Forecasting \& Social Change, Año 77, pp. 369-384.

Hempell, Thomas. (2003), Do computers call for Training? Firm-level Evidence on Complementarities between ICT and Human Capital Investments, ZEN Discussion Papers, No 03-20, Mannheim.

López Sánchez, José Luis, (2004) ¿Pueden las Tecnologías de la Información mejorar la productividad? Universia Business Review, Año. 1, pp. 82-95.

Nieto, María Jesús y Fernandez, Zulima (2006), The role of information technology in business strategy of small and medium enterprises, Journal of International Entrepreneurs, Año 3, pp. 251-262. 
OCDE (2000), Perspectives économiques de I'OCDE: Quels sont les facteurs qui sous-tendent la croissance dans les différents pays de I 'OCDE? No 67.

Oliveira, Tiago y Fraga Martins, Maria, (2011), Literature Review of Information Technology adoption models at firm level. The Electronic Journal of Information Systems Evaluation, Año 14, No 1, pp. 110-121.

Pérez Estébanez, Raquel (2012), Las Tecnologías de la Información y la Comunicación (TIC) se consolidan en las empresas españolas a pesar de la crisis económica: Análisis comparativo entre 2005 y 2009. Cuadernos de Contabilidad, 13 (33), 479-503.

Pérez, Raquel, Urquía, Elena y Muñoz, Clara, (2010), Information technology implementation: Evidence in spanish SMEs. International Journal of Accounting and Information Management, Año 18, No 1, pp. 39-57.

Ravichandran, Thiagarajan, (2005), Effect of Information Systems, Resources and Capabilities on Firm Performance: A resource based perspective, Journal of Management Information Systems, Año 21, pp. 237-276.

Rhee, Jay Hyuk, (2005), The Internet era and the international expansion process: The moderating role of absorptive capacity, Management International Review, Año 45, No 3, pp. 277-306.

Ríos, Martha, López, Alejandra y Contreras, Ricardo, (2013), Reconocimiento y Compromiso de las TIC en las empresas del Estado de Guanajuato. Revista Panorama Administrativo, Año 7, No 13, pp. 5-24.

Salazar, Mauren, Arzola, Minerva. y Pérez, Elvira, (2010), Gestión de la innovación para las Pymis de Ciudad Guayana, Revista Venezolana de Gerencia, Año 15 No 51, pp. 446-461.
Santana Ormeño, Martín y Aspilcueta Loayza, Heidi, (2015), Prioridades de gestión de tecnologías de información en organizaciones peruanas. Revista Venezolana de Gerencia, Año. 20, No. 72, pp. 684-697.

Scapens, Robert y Jazayeri, Mostafa (2003), ERP Systems and Management Accounting change: Opportunities or Impacts? A research note, European Accounting Review, Año 12, No 1, pp. 201-233.

Scapens, Robert, y Jazayeri, Mostafa (1998), SAP: integrated Information Systems and the implications for Management Accountants, Management Accounting, Año 76, No 8, pp. 46-48.

Tushman, Michael y Anderson, Phillip. (1986), Technological discontinuities and organizational environments, Administrative Science Quarterly, Año 31, pp. 439-465.

Van de Ven, Andrew y Drazin, Robert, (1985), The concept of fit in contingency theory, Resources Organizational Behaviour, Año 7, pp. 65-333.

Wolf, Elke y Zwick, Thomas, (2002), Reassessing the Impact of High Performance Workplaces, ZEW Discussion Paper $n^{\circ}$ 02-07, Revised Version, Manheim.

World Bank (2006): Information and Communications for Development 2006: Global Trends and Policies, THE WORLD BANK. Recuperado el 1 de Mayo de 2016, de http:// siteresources.worldbank.org/E XTINF ORMATIONANDCOMMUNICATIONA NDTECHNOLOGIES/Resources/282 822-1141851022286/IC4DOverview. pdf 


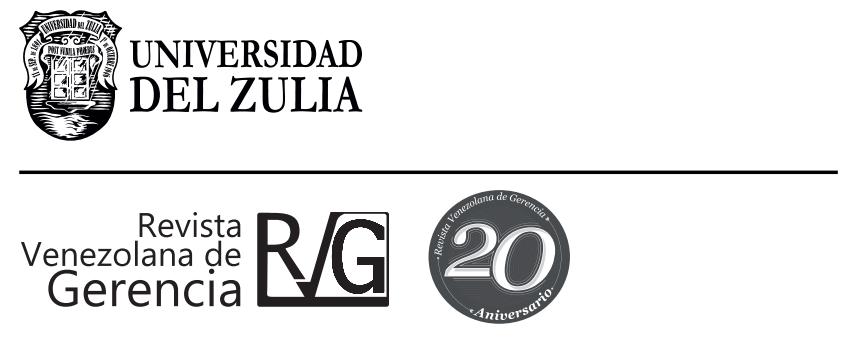

Año 21, No. 74

Esta revista fue editada en formato digital y publicada en junio del 2016, por la Universidad del Zulia, Vicerrectorado Académico, Serbiluz - Fondo editorial, Maracaibo -Venezuela. 